\title{
Espaçamento de plantio e intervalos de colheita na biomassa e no óleo essencial de gerânio
}

\author{
Arie F Blank; Anderson de C Silva; Maria de Fátima Arrigoni-Blank; Wallace M dos Santos; Aléa \\ Dayane D de Santana \\ UFS-DEA, Av. Marechal Rondon s/n, 49100-000 São Cristóvão-SE; afblank@ufs.com; bio.anderson@gmail.com; arrigoni@ufs.br; \\ wallacemds@hotmail.com aleadayane@gmail.com
}

\section{RESUMO}

O gerânio (Pelargonium graveolens) é uma erva aromática e seu óleo essencial é comumente utilizado na constituição de fármacos e cosméticos no mundo. A planta adapta-se bem às condições climáticas do nordeste brasileiro, mas são escassos os dados sobre seu rendimento quantitativo e qualitativo. O objetivo do trabalho foi avaliar a influência de espaçamento de plantas e intervalos de colheita em gerânio na produção de biomassa e de óleo essencial. Foram testados três espaçamentos de plantio (50x50, 50x60 e 50x80 $\mathrm{cm})$ e três intervalos de colheita (8,12 e 16 semanas). Os maiores valores totais de massa fresca e seca de folhas e caules $(2679,04 \mathrm{~g}$ $\mathrm{m}^{-2} ; 424,62 \mathrm{~g} \mathrm{~m}^{-2} ; 1035,08 \mathrm{~g} \mathrm{~m}^{-2} ; 136,85 \mathrm{~g} \mathrm{~m}^{-2}$, respectivamente) $\mathrm{e}$ rendimento de óleo essencial $\left(7,56 \mathrm{~mL} \mathrm{~m}^{-2}\right)$, que são características de interesse direto para o mercado, foram obtidos em intervalo de colheita de oito semanas e no espaçamento de $50 \times 50 \mathrm{~cm}$. Nas demais variáveis analisadas, houve pouca diferenciação entre os tratamentos, contudo, períodos longos de colheita são menos produtivos, pois houve queda nos valores de todas as variáveis nas ultimas colheitas de cada intervalo. Assim, melhores resultados de quantidade e qualidade para o gerânio foram obtidos com intervalo de 8 semanas e no espaçamento $50 \times 50 \mathrm{~cm}$.

Palavras-chave: Pelargonium graveolens, planta aromática, cultivo.

\begin{abstract}
Plant spacing and harvest intervals on biomass and essential oil of geranium

Geranium (Pelargonium graveolens) is an aromatic herb and its essential oil is commonly used in the creation of drugs and cosmetics worldwide. The plant is well adapted to the climatic conditions of the Brazilian northeast, but there are few data on its quantitative and qualitative yield. The aim of this study was to evaluate the influence of plant spacing and harvest intervals of geranium on the production of biomass and essential oil. Three different plant spacings (50x50, 50x60 and 50x80 cm) and three harvest intervals (8, 12 and 16 weeks) were tested. The highest values of total fresh and dry weight of leaves and stems (2679.04 $\mathrm{g} \mathrm{m}^{-2} ; 424.62 \mathrm{~g} \mathrm{~m}^{-2} ; 1035.08 \mathrm{~g} \mathrm{~m}^{-2} ; 136.85 \mathrm{~g}$ $\mathrm{m}^{-2}$, respectively) and yield of essential oil $\left(7.56 \mathrm{~mL} \mathrm{~m}^{-2}\right)$, that are characteristics of direct interest for the market, were obtained at harvest intervals of eight weeks and at a spacing of 50x $50 \mathrm{~cm}$. In other analyzed variables, there was little difference between the treatments, however, long periods of harvest are less productive because decrease of values of all variables were observed in the last harvests of each interval. Thus, best quantity and quality results for geranium were obtained on an interval of 8 weeks, and spacing of $50 \times 50 \mathrm{~cm}$.
\end{abstract}

Keywords: Pelargonium graveolens, aromatic plant, cultivation.

\section{(Recebido para publicação em 31 de agosto de 2011; aceito em 13 de novembro de 2012) (Received on August 31, 2011; accepted on November 13, 2012)}

$\mathrm{O}$ Pelargonium graveolens é uma erva medicinal e aromática, nativa do sul da África. Sua importância reside principalmente no valor aromático do óleo essencial armazenado em células do caule, folhas e flores (Saxena et al., 2000; Lis-Balchin, 2002; Rana et al., 2003).Compostos voláteis presentes no óleo essencial de gerânio, principalmente geraniol, citronelol e linalol, são de amplo emprego nas indústrias de perfumaria, cosméticos, sabões, alimentícia e de aromáticos. Seu óleo é também utilizado na medicina popular no combate a um vasto número de doenças (Peterson et al., 2006).

Os materiais comerciais de gerânio podem ser classificados em três tipos: Algério, Bourbon e Egípcio. Para di- ferenciar quimicamente os três tipos, deve-se observar o teor dos sequiterpenos 10-epi- $\gamma$-eudesmol e 6,9-guiadieno (Kaul et al., 1997; Ram et al., 1997; Rao et al., 2002).

A produção mundial de óleo essencial de gerânio de aproximadamente 600 toneladas ( $\mathrm{t}$ ) vem sendo alcançada pela China, Marrocos, Egito, Ilha Reunião e África do Sul (Ram et al., 2003). Ainda assim, há um déficit de $200 \mathrm{t}$ de óleo essencial de gerânio por ano. Por isso, torna-se necessário ampliar o volume de produção dessa cultura, principalmente em regiões propícias à sua adaptação como o Brasil.

Trabalhos sobre a aplicação de tratos culturais vêm sendo realizados, visando a domesticação de espécies exóticas na tentativa de suprir as demandas de produção de algumas culturas. O foco dessas pesquisas é, geralmente, o plantio, a colheita, o processamento e a extração do óleo essencial, onde é avaliada a influência de cada época de desenvolvimento na produtividade da cultura (Gomes et al., 2004).

Atualmente, é conhecida a influência da densidade de plantio sobre o desenvolvimento vegetal. Os estudos comprovam que o espaçamento entre plantas e entre linhas alteram o desenvolvimento das plantas em diferentes níveis (Bergo et al., 2005; Puquerio et al., 2007). Em trabalhos com plantas medicinais e aromáticas, essas alterações também são reportadas para a biossíntese dos óleos essenciais, podendo alterar seu rendi- 
mento e composição (Badi et al., 2004; Missaoui et al., 2005; Mighri et al., 2009). Na Índia, o cultivo comercial de gerânio é feito utilizando, preferencialmente, o espaçamento de $50 \mathrm{~cm}$ entre linhas x $50 \mathrm{~cm}$ entre plantas (Kothari et al., 2002).

Outro fator tão importante quanto o espaçamento para definir a produtividade total de uma cultura é o intervalo de tempo entre as colheitas. O rendimento de biomassa e de óleo essencial da planta pode ser modificado em função dos fatores climáticos na época da colheita. Aliado a isso, o tempo de rebrota pode influenciar os valores reais de massa fresca e seca, assim como o rendimento e teor dos óleos essenciais. Em estudo realizado com a espécie medicinal Tomilho (Thymus vulgaris), testando colheitas em três períodos (inicio da floração; plena floração; pós floração) foi verificado que as colheitas realizadas em menor tempo de desenvolvimento foram mais rentáveis, tanto para biomassa quanto para rendimento e teor de óleo essencial (Badi et al., 2004).

Considerando a escassez de informações sobre o gerânio no Brasil sobre seu manejo cultural e a sua relevância, o objetivo foi avaliar a influência do espaçamento e do intervalo de colheitas sobre a produção de biomassa, o rendimento e o teor do óleo essencial de gerânio (Pelargonium graveolens).

\section{MATERIAL E MÉTODOS}

O experimento de campo teve duração de um ano e foi conduzido entre os anos de 2008 a 2010, na Fazenda Experimental "Campus Rural da UFS", São Cristóvão a $17 \mathrm{~km}$ de Aracaju. Foram utilizadas plantas do genótipo UFS-PEL001 de gerânio (tipo Egípcio), mantidas no Banco Ativo de Germoplasma da Universidade Federal de Sergipe. Exsicatas do genótipo foram depositadas no Herbário da Universidade Federal de Sergipe (ASE) sob o número de registro ASE-14844.

O solo da área é do tipo argissolo vermelho-amarelo distrófico, de textura franco-arenosa com as seguintes características químicas: $\mathrm{pH}=4,6$; matéria orgânica $=21,2 \mathrm{~g} \mathrm{dm}^{-3} ; 23,3$ ppm de P;
34,9 ppm de K; 7,9 ppm de $\mathrm{Na} ; 1,19$ cmolc/dm ${ }^{3}$ de $\mathrm{Ca} ; 1,51 \mathrm{cmolc} / \mathrm{dm}^{3}$ de $\mathrm{Mg} ; 4,85 \mathrm{cmolc} / \mathrm{dm}^{3}$ de CTC e 58,1\% V.

$\mathrm{O}$ experimento foi instalado em delineamento de blocos ao acaso, em esquema de parcelas subdivididas no tempo, com três repetições. Foram testados, nas parcelas, três combinações de espaçamentos entre linhas e entre fileiras $(50 \times 50 ; 50 \times 60$ e 50x80 cm) e, nas subparcelas, três intervalos de colheita (8, 12 e 16 semanas). Cada subparcela foi formada por oito plantas, sendo as quatro centrais consideradas parcela útil. Para o intervalo de colheita de oito semanas, foram efetuados sete cortes, para o intervalo de 12 semanas, cinco cortes, e para o intervalo de 16 semanas, apenas quatro cortes. A variação no número de cortes foi devido ao limite de tempo considerado para a avaliação do experimento, sendo possível sete colheitas sobre o intervalo de oito semanas, enquanto o de 16 semanas só foi possível a realização de quatro colheitas.

As plantas de gerânio foram propagadas por meio de estaquia. As estacas foram retiradas das porções apicais dos ramos, com $10 \mathrm{~cm}$ cada, colocadas em sacos plásticos com capacidade de 1,5 L, contendo a mistura solo: areia, na proporção $1: 1$. As estacas foram assim mantidas por 45 dias em estufa plástica protegida com tela de $50 \%$ de sombreamento, sendo posteriormente transplantadas para o campo.

Os ensaios foram implantados em dezembro de 2008 e a primeira colheita em fevereiro de 2009. Foi utilizada irrigação por gotejamento, adubação de $20 \mathrm{t} \mathrm{ha}^{-1}$ de esterco bovino combinado com $1.000 \mathrm{~kg} \mathrm{ha}^{-1}$ da fórmula 3-12-6 e cobertura do solo com filme plástico de cor prata; essa cobertura foi utilizada visando favorecer o microclima do solo e diminuir a ação de invasoras sobre as plantas de gerânio. Foram realizadas capinas e limpeza dos canteiros durante o experimento.

As variáveis analisadas a cada colheita foram altura das plantas (avaliadas desde a base até a ultima folha do ramo mais alto), diâmetro de parte aérea, massa fresca e seca de folhas e caules, teor e rendimento de óleo essencial.

Para a avaliação da massa fresca, os cortes na colheita foram feitos à altura de aproximadamente $20 \mathrm{~cm}$ do solo. Foram separadas as folhas e caules da parte aérea colhida, que foram estratificadas por tratamento e por bloco, sendo pesadas. Para determinar a massa seca foram usadas três amostras de 100 gramas de folha fresca e colocadas em estufa de secagem a $105^{\circ} \mathrm{C}$ por 24 horas, seguida de pesagem.

O óleo essencial foi obtido das folhas frescas, por meio do processo de hidrodestilação, por duas horas e 40 minutos, com a utilização do aparelho de Clevenger (Guenther, 1972). Os teores (\%) foram estimados com base no peso da matéria seca (v/m) e obtidos usando três amostras de 100 gramas.

As variáveis analisadas foram submetidas à análise de variância pelo teste de F e comparadas pelo teste de Scott-Knott a $5 \%$ de probabilidade.

\section{RESULTADOS E DISCUSSÃO}

Não houve interação significativa entre os intervalos de corte e os espaçamentos para as variáveis avaliadas, sendo as comparações feitas independentemente em cada colheita (Tabela 1 a 4).

$\mathrm{Na}$ colheita feita com oito semanas, as plantas de gerânio apresentaram significativa redução no desenvolvimento após a quarta e quinta colheitas, exceto para diâmetro e teor e rendimento de óleo essencial (Tabela 1). Comparando-se os valores médios de altura da primeira e ultima colheitas, houve uma redução de 53, 61 e 48,7\% quando foram utilizados os espaçamentos de 50x50, $50 \times 60$ e 50x80 cm, respectivamente. Os resultados apresentados demonstram que no início do cultivo as plantas não foram afetadas pelo menor espaçamento e, consequentemente competição entre elas, mas no menor espaçamento isso limitou o crescimento em altura a partir da quarta colheita.

Houve redução também, do percentual de sobrevivência entre as colheitas em função dos espaçamentos. Na última colheita os valores foram 58,33\% para o espaçamento $50 \times 50 \mathrm{~cm}, 25 \%$ para o espaçamento de $50 \times 60 \mathrm{~cm}$ e $33,33 \%$ para o espaçamento de $50 \times 80 \mathrm{~cm}$. Esse resultado comprometeu, sobretudo, a avaliação das demais variáveis, desencadeando um processo cascata, levando à redução em até $76 \%$ para as variáveis, 
Tabela 1. Altura de planta $(\mathrm{cm})$, diâmetro de parte aérea $(\mathrm{cm})$, sobrevivência $(\%)$, massa fresca e seca de folhas e caules e teor e rendimento de óleo essencial na produção de gerânio (P. graveolens), realizando colheitas a cada oito semanas durante um período de um ano (plant height $(\mathrm{cm})$, canopy diameter $(\mathrm{cm})$, survival (\%), fresh and dry weight of leaves and stems, and content and yield of essential oil in the production of geranium (P. graveolens) and harvest carried out every eight weeks over a period of one year). São Cristóvão, UFS, 2011.

\begin{tabular}{|c|c|c|c|c|c|c|}
\hline \multirow{2}{*}{ № da colheita } & \multicolumn{3}{|c|}{ Espaçamento (cm) } & \multicolumn{3}{|c|}{ Espaçamento $(\mathrm{cm})$} \\
\hline & $50 \times 50$ & $50 \times 60$ & $50 \times 80$ & $50 \times 50$ & $50 \times 60$ & $50 \times 80$ \\
\hline & \multicolumn{3}{|c|}{ Altura de planta $(\mathrm{cm})$} & \multicolumn{3}{|c|}{ Massa fresca de caules (g m-2) } \\
\hline$\overline{1^{\underline{a}}}$ & $66,97 \mathrm{aA}$ & $70,86 \mathrm{aA}$ & $63,86 \mathrm{aA}$ & $426,66 \mathrm{aA}$ & $261,11 \mathrm{aB}$ & $241,66 \mathrm{aB}$ \\
\hline $2^{\mathrm{a}}$ & $53,55 \mathrm{aA}$ & $54,24 \mathrm{aA}$ & $55,33 \mathrm{aA}$ & $232,22 \mathrm{bA}$ & $183,33 \mathrm{aA}$ & $136,11 \mathrm{aA}$ \\
\hline $3^{\mathrm{a}}$ & $56,16 \mathrm{aA}$ & $57,46 \mathrm{aA}$ & $60,54 \mathrm{aA}$ & $110,90 \mathrm{bA}$ & $103,97 \mathrm{bA}$ & $98,85 \mathrm{aA}$ \\
\hline $4^{\mathrm{a}}$ & $51,25 \mathrm{aA}$ & $52,83 \mathrm{aA}$ & $50,66 \mathrm{aA}$ & $113,33 \mathrm{bA}$ & $53,24 \mathrm{bA}$ & $34,72 \mathrm{aA}$ \\
\hline $5^{\mathrm{a}}$ & $52,41 \mathrm{aA}$ & $38,50 \mathrm{bA}$ & $45,83 \mathrm{bA}$ & $73,06 \mathrm{bA}$ & $55,80 \mathrm{bA}$ & $89,60 \mathrm{aA}$ \\
\hline $6^{\mathrm{a}}$ & $41,12 \mathrm{bA}$ & $35,75 \mathrm{bA}$ & $44,58 \mathrm{bA}$ & $55,00 \mathrm{bA}$ & $41,66 \mathrm{bA}$ & $26,04 \mathrm{aA}$ \\
\hline \multirow[t]{2}{*}{$7 \mathrm{a}$} & $31,41 \mathrm{bA}$ & $27,50 \mathrm{bA}$ & $32,75 \mathrm{bA}$ & $63,33 \mathrm{bA}$ & $25,00 \mathrm{bA}$ & $65,62 \mathrm{aA}$ \\
\hline & \multicolumn{3}{|c|}{ Diâmetro de parte aérea $(\mathrm{cm})$} & \multicolumn{3}{|c|}{ Massa seca de caules (g m-2) } \\
\hline $1 \underline{\underline{a}}$ & $70,45 \mathrm{aA}$ & $75,78 \mathrm{aA}$ & $74,46 \mathrm{aA}$ & $78,11 \mathrm{aA}$ & $36,70 \mathrm{aB}$ & $38,39 \mathrm{aB}$ \\
\hline $2^{\mathrm{a}}$ & $55,69 \mathrm{aA}$ & $60,31 \mathrm{aA}$ & $65,18 \mathrm{aA}$ & $63,58 \mathrm{aA}$ & $30,94 \mathrm{aA}$ & $21,89 \mathrm{aA}$ \\
\hline $3^{\mathrm{a}}$ & $68,21 \mathrm{aA}$ & $68,91 \mathrm{aA}$ & $74,18 \mathrm{aA}$ & $39,93 \mathrm{bA}$ & $15,34 \mathrm{aA}$ & $14,62 \mathrm{aA}$ \\
\hline $4^{\mathrm{a}}$ & $71,41 \mathrm{aA}$ & $63,75 \mathrm{aA}$ & $61,89 \mathrm{aA}$ & $15,94 \mathrm{bA}$ & $8,75 \mathrm{aA}$ & $5,84 \mathrm{aA}$ \\
\hline 5 a & $63,83 \mathrm{aA}$ & $50,50 \mathrm{aA}$ & $59,41 \mathrm{aA}$ & $17,38 \mathrm{bA}$ & $12,94 \mathrm{aA}$ & $20,43 \mathrm{aA}$ \\
\hline $6^{\mathrm{a}}$ & $55,71 \mathrm{aA}$ & $64,33 \mathrm{aA}$ & $66,58 \mathrm{aA}$ & $17,13 \mathrm{bA}$ & $6,25 \mathrm{aA}$ & $4,51 \mathrm{aA}$ \\
\hline \multirow[t]{2}{*}{7 a } & $41,66 \mathrm{aA}$ & $48,25 \mathrm{aA}$ & $55,00 \mathrm{aA}$ & $10,74 \mathrm{bA}$ & $4,60 \mathrm{aA}$ & $16,71 \mathrm{aA}$ \\
\hline & \multicolumn{3}{|c|}{ Sobrevivência (\%) } & \multicolumn{3}{|c|}{$\begin{array}{l}\text { Teor de óleo essencial }(\%) \\
\end{array}$} \\
\hline$\overline{1^{\underline{a}}}$ & $100,00 \mathrm{aA}$ & $91,66 \mathrm{aA}$ & $83,33 \mathrm{aA}$ & $1,33 \mathrm{aA}$ & $1,28 \mathrm{aA}$ & $1,31 \mathrm{aA}$ \\
\hline $2^{\mathrm{a}}$ & $91,66 \mathrm{aA}$ & $91,66 \mathrm{aA}$ & $75,00 \mathrm{aA}$ & $1,33 \mathrm{aA}$ & $1,15 \mathrm{aA}$ & $1,27 \mathrm{aA}$ \\
\hline $3^{\mathrm{a}}$ & $100,00 \mathrm{aA}$ & $100,00 \mathrm{aA}$ & $100,00 \mathrm{aA}$ & $1,67 \mathrm{aA}$ & $1,37 \mathrm{aA}$ & $1,36 \mathrm{aA}$ \\
\hline $4^{\mathrm{a}}$ & $83,33 \mathrm{aA}$ & $83,33 \mathrm{aA}$ & $75,00 \mathrm{aA}$ & $1,67 \mathrm{aA}$ & $1,59 \mathrm{aA}$ & $1,31 \mathrm{aA}$ \\
\hline 5 a & $83,33 \mathrm{aA}$ & $66,66 \mathrm{aA}$ & $75,00 \mathrm{aA}$ & $1,35 \mathrm{aA}$ & $1,21 \mathrm{aA}$ & $1,60 \mathrm{aA}$ \\
\hline $6^{\mathrm{a}}$ & $58,33 \mathrm{bA}$ & $41,66 \mathrm{bA}$ & $41,66 \mathrm{bA}$ & $1,14 \mathrm{aA}$ & $1,36 \mathrm{aA}$ & $1,09 \mathrm{aA}$ \\
\hline \multirow[t]{2}{*}{$7 \mathrm{a}$} & $41,66 \mathrm{bA}$ & $25,00 \mathrm{bA}$ & $33,33 \mathrm{bA}$ & $1,26 \mathrm{aA}$ & $1,36 \mathrm{aA}$ & $1,18 \mathrm{aA}$ \\
\hline & \multicolumn{3}{|c|}{ Massa fresca de folhas $\left(\mathrm{g} \mathrm{m}^{-2}\right)$} & \multicolumn{3}{|c|}{ Rendimento de óleo essencial (mL m-2) } \\
\hline 1"a & $893,33 \mathrm{aA}$ & $543,52 \mathrm{aB}$ & $572,91 \mathrm{aB}$ & $2,24 \mathrm{aA}$ & $1,21 \mathrm{aB}$ & $1,43 \mathrm{aB}$ \\
\hline $2^{\mathrm{a}}$ & $543,33 \mathrm{bA}$ & $557,40 \mathrm{aA}$ & $393,05 \mathrm{aA}$ & $1,48 \mathrm{aA}$ & $1,37 \mathrm{aA}$ & $0,97 \mathrm{aA}$ \\
\hline 3 a & $367,71 \mathrm{bA}$ & $310,76 \mathrm{bA}$ & $320,06 \mathrm{aA}$ & $1,26 \mathrm{aA}$ & $0,76 \mathrm{aA}$ & $0,83 \mathrm{aA}$ \\
\hline $4^{\mathrm{a}}$ & $363,33 \mathrm{bA}$ & $176,85 \mathrm{bA}$ & $129,86 \mathrm{aA}$ & $1,12 \mathrm{aA}$ & $0,53 \mathrm{aB}$ & $0,35 \mathrm{aB}$ \\
\hline $5^{\mathrm{a}}$ & $189,10 \mathrm{bA}$ & $138,94 \mathrm{bA}$ & $207,48 \mathrm{aA}$ & $0,50 \mathrm{aA}$ & $0,33 \mathrm{aA}$ & $0,74 \mathrm{aA}$ \\
\hline $6^{\mathrm{a}}$ & $200,00 \mathrm{bA}$ & $141,67 \mathrm{bA}$ & $137,50 \mathrm{aA}$ & $0,58 \mathrm{aA}$ & $0,34 \mathrm{aA}$ & $0,33 \mathrm{aA}$ \\
\hline $7 \underline{\mathrm{a}}$ & $283,33 \mathrm{bA}$ & $150,00 \mathrm{bA}$ & $137,50 \mathrm{aA}$ & $0,82 \mathrm{aA}$ & $0,45 \mathrm{aA}$ & $0,36 \mathrm{aA}$ \\
\hline
\end{tabular}

\begin{tabular}{|c|c|c|c|c|}
\hline \multicolumn{4}{|c|}{ Massa seca de folhas $\left(\mathrm{g} \mathrm{m}^{-2}\right)$} & \\
\hline $1^{\mathrm{a}}$ & $179,49 \mathrm{aA}$ & $107,91 \mathrm{aB}$ & $110,30 \mathrm{aB}$ & \\
\hline $2^{\mathrm{a}}$ & $106,81 \mathrm{bA}$ & $110,77 \mathrm{aA}$ & 81,26 aA & \\
\hline $3^{\mathrm{a}}$ & $73,28 \mathrm{bA}$ & $58,43 \mathrm{bA}$ & $62,45 \mathrm{aA}$ & \\
\hline $4^{\mathrm{a}}$ & $65,02 \mathrm{bA}$ & $33,12 \mathrm{bA}$ & $24,99 \mathrm{aA}$ & \\
\hline $5 \mathrm{a}$ & $37,86 \mathrm{bA}$ & $28,36 \mathrm{bA}$ & $43,08 \mathrm{aA}$ & \\
\hline $6^{\mathrm{a}}$ & $46,40 \mathrm{bA}$ & $26,33 \mathrm{bA}$ & $30,87 \mathrm{aA}$ & \\
\hline 7 a & $55,90 \mathrm{bA}$ & $30,25 \mathrm{bA}$ & $29,05 \mathrm{aA}$ & \\
\hline
\end{tabular}

Médias seguidas pelas mesmas letras, minúsculas nas colunas e maiúsculas nas linhas, não diferem entre si pelo teste de Scott-Knott (means followed by same letters, uppercase in lines and lowercase in columns do not differ by Scott-Knott test $)(\mathrm{p} \leq 0,05)$. 
Tabela 2. Altura de planta $(\mathrm{cm})$, diâmetro de parte aérea $(\mathrm{cm})$, sobrevivência $(\%)$, massa fresca e seca de folhas e caules e, teor e rendimento de óleo essencial na produção de gerânio (P. graveolens), realizando colheitas a cada 12 semanas durante um período de um ano (plant height $(\mathrm{cm})$, canopy diameter $(\mathrm{cm})$, survival (\%), fresh and dry weight of leaves and stems, and content and yield of essential oil production of geranium (P. graveolens) and harvests carried out every 12 weeks over a period of one year). São Cristóvão, UFS, 2011.

\begin{tabular}{|c|c|c|c|c|c|c|}
\hline \multirow{2}{*}{ Colheita } & \multicolumn{3}{|c|}{ Espaçamento $(\mathrm{cm})$} & \multicolumn{3}{|c|}{ Espaçamento (cm) } \\
\hline & $50 \times 50$ & $50 \times 60$ & $50 \times 80$ & $50 \times 50$ & $50 \times 60$ & $50 \times 80$ \\
\hline & \multicolumn{3}{|c|}{ Altura de planta $(\mathrm{cm})$} & \multicolumn{3}{|c|}{ Massa fresca de caule (g m-2) } \\
\hline $1^{\mathrm{a}}$ & $73,35 \mathrm{aA}$ & $55,78 \mathrm{aA}$ & $61,72 \mathrm{aA}$ & 303,93 aA & $211,11 \mathrm{aB}$ & $170,83 \mathrm{aB}$ \\
\hline $2^{\mathrm{a}}$ & $71,61 \mathrm{aA}$ & $53,44 \mathrm{aA}$ & $65,42 \mathrm{aA}$ & $282,22 \mathrm{aA}$ & $209,25 \mathrm{aA}$ & $207,57 \mathrm{aA}$ \\
\hline $3^{\mathrm{a}}$ & $78,94 \mathrm{aA}$ & $66,00 \mathrm{aA}$ & $77,16 \mathrm{aA}$ & $290,55 \mathrm{aA}$ & $143,52 \mathrm{aB}$ & $195,14 \mathrm{aB}$ \\
\hline $4^{\mathrm{a}}$ & $58,58 \mathrm{aA}$ & $48,25 \mathrm{aA}$ & $59,99 \mathrm{aA}$ & $176,66 \mathrm{aA}$ & $66,66 \mathrm{aB}$ & $88,89 \mathrm{aB}$ \\
\hline \multirow[t]{2}{*}{ 5a } & $63,75 \mathrm{aA}$ & $48,94 \mathrm{aA}$ & $64,39 \mathrm{aA}$ & 256,66 aA & $115,74 \mathrm{aB}$ & $143,05 \mathrm{aB}$ \\
\hline & \multicolumn{3}{|c|}{ Diâmetro de parte aérea $(\mathrm{cm})$} & \multicolumn{3}{|c|}{ Massa seca de caule (g m-2) } \\
\hline $1^{\mathrm{a}}$ & $75,01 \mathrm{aA}$ & $59,74 \mathrm{aB}$ & $73,10 \mathrm{aA}$ & $43,19 \mathrm{aA}$ & $32,14 \mathrm{aA}$ & $27,60 \mathrm{aA}$ \\
\hline $2^{\mathrm{a}}$ & 76,66 aA & $57,88 \mathrm{aB}$ & $83,00 \mathrm{aA}$ & $41,10 \mathrm{aA}$ & $29,20 \mathrm{aA}$ & $31,52 \mathrm{aA}$ \\
\hline $3^{\mathrm{a}}$ & $97,41 \mathrm{aA}$ & $82,44 \mathrm{aB}$ & $98,11 \mathrm{aA}$ & $47,91 \mathrm{aA}$ & $24,29 \mathrm{aB}$ & $32,49 \mathrm{aB}$ \\
\hline $4^{\mathrm{a}}$ & $92,16 \mathrm{aA}$ & $71,28 \mathrm{aB}$ & $85,11 \mathrm{aA}$ & $38,26 \mathrm{aA}$ & $16,34 \mathrm{aB}$ & $19,73 \mathrm{aB}$ \\
\hline \multirow[t]{2}{*}{$5^{\mathrm{a}}$} & $91,50 \mathrm{aA}$ & $73,58 \mathrm{aA}$ & $82,05 \mathrm{aA}$ & $49,11 \mathrm{aA}$ & $25,23 \mathrm{aB}$ & $28,08 \mathrm{aB}$ \\
\hline & \multicolumn{3}{|c|}{ Sobrevivência (\%) } & \multicolumn{3}{|c|}{ Teor de óleo essencial $(\%)$} \\
\hline $1^{\mathrm{a}}$ & $100,00 \mathrm{aA}$ & $100,00 \mathrm{aA}$ & $100,00 \mathrm{aA}$ & $1,33 \mathrm{aA}$ & $1,28 \mathrm{aA}$ & $1,31 \mathrm{aA}$ \\
\hline $2^{\underline{a}}$ & $91,66 \mathrm{aA}$ & $83,33 \mathrm{aA}$ & $91,66 \mathrm{aA}$ & $1,25 \mathrm{aA}$ & $1,44 \mathrm{aA}$ & $1,34 \mathrm{aA}$ \\
\hline $3^{\mathrm{a}}$ & $91,66 \mathrm{aA}$ & $83,33 \mathrm{aA}$ & $91,66 \mathrm{aA}$ & $1,37 \mathrm{aA}$ & $1,31 \mathrm{aA}$ & $1,38 \mathrm{aA}$ \\
\hline $4^{\mathrm{a}}$ & $41,66 \mathrm{bB}$ & $83,33 \mathrm{aA}$ & $75,00 \mathrm{bA}$ & $1,11 \mathrm{aA}$ & $0,93 \mathrm{aA}$ & $1,09 \mathrm{aA}$ \\
\hline \multirow[t]{2}{*}{$5^{\mathrm{a}}$} & $41,66 \mathrm{bB}$ & $83,33 \mathrm{aA}$ & $58,33 \mathrm{bB}$ & $1,52 \mathrm{aA}$ & $1,08 \mathrm{aA}$ & $1,28 \mathrm{aA}$ \\
\hline & \multicolumn{3}{|c|}{ Massa fresca de folhas $\left(\mathrm{g} \mathrm{m}^{-2}\right)$} & \multicolumn{3}{|c|}{ Rendimento de óleo essencial (mL m-2) } \\
\hline$\overline{1}$ & $684,46 \mathrm{aA}$ & $464,81 \mathrm{aB}$ & $441,66 \mathrm{aB}$ & $1,79 \mathrm{aA}$ & $1,21 \mathrm{aB}$ & $1,12 \mathrm{aB}$ \\
\hline $2^{\mathrm{a}}$ & $765,55 \mathrm{aA}$ & $593,51 \mathrm{aA}$ & $610,40 \mathrm{aA}$ & $1,81 \mathrm{aA}$ & $1,52 \mathrm{aA}$ & $1,66 \mathrm{aA}$ \\
\hline $3^{-\mathbf{a}}$ & $674,44 \mathrm{aA}$ & $346,29 \mathrm{bB}$ & $464,55 \mathrm{aB}$ & $1,78 \mathrm{aA}$ & $0,84 \mathrm{aB}$ & $1,27 \mathrm{aB}$ \\
\hline $4^{\mathrm{a}}$ & 546,66 aA & $153,70 \mathrm{bB}$ & $238,88 \mathrm{bB}$ & $1,45 \mathrm{aA}$ & $0,32 \mathrm{aB}$ & $0,54 \mathrm{aB}$ \\
\hline \multirow[t]{2}{*}{$5^{\mathrm{a}}$} & $573,33 \mathrm{aA}$ & $250,92 \mathrm{bB}$ & $266,66 \mathrm{bB}$ & $1,72 \mathrm{aA}$ & $0,54 \mathrm{aB}$ & $0,69 \mathrm{aB}$ \\
\hline & \multicolumn{3}{|c|}{ Massa seca de folhas (g m-2) } & & & \\
\hline $1^{\mathrm{a}}$ & $136,44 \mathrm{aA}$ & $94,76 \mathrm{aB}$ & $86,64 \mathrm{aB}$ & & & \\
\hline $2^{\mathrm{a}}$ & $143,33 \mathrm{aA}$ & $106,94 \mathrm{aA}$ & $122,28 \mathrm{aA}$ & & & \\
\hline $3^{-}$ & $120,75 \mathrm{aA}$ & $65,26 \mathrm{bB}$ & $89,35 \mathrm{aB}$ & & & \\
\hline $4^{\mathrm{a}}$ & $121,93 \mathrm{aA}$ & $34,93 \mathrm{bB}$ & $55,56 \mathrm{aB}$ & & & \\
\hline 5 & $108,88 \mathrm{aA}$ & $51,76 \mathrm{bB}$ & $53,59 \mathrm{aB}$ & & & \\
\hline
\end{tabular}

Médias seguidas pelas mesmas letras, minúsculas nas colunas e maiúsculas nas linhas, não diferem entre si pelo teste de Scott-Knott (means followed by same letters, uppercase in lines and lowercase in columns do not differ by Scott-Knott test) $(\mathrm{p} \leq 0,05)$.

massa fresca e seca de folhas e caules, em relação aos espaçamentos 50x50 e $50 \times 60 \mathrm{~cm}$. Contudo, essas variáveis não apresentaram diferenças significativas, entre os cortes, em relação ao espaçamento 50x80 cm (Tabela 1). Esses números demonstram que as plantas de gerânio não resistem aos sucessivos cortes em intervalos pequenos durante um longo período de tempo, levando à perda de rendimento ao longo do ano mas, mesmo assim, o rendimento total de óleo essencial justifica a utilização do espaçamento de oito semanas (Tabela 4).

Ainda, em relação à colheita em oito semanas, os espaçamentos não diferiram em altura, diâmetro e porcentagem de sobrevivência (Tabela 1). Entretanto, maior produção de biomassa e rendimento de óleo essencial no primeiro corte foram obtidos para o espaçamento de 50x50 cm em relação aos demais espaçamentos (Tabela 1). Isso é consequência da densidade de plantio, pois não houve variação entre os espaçamentos para as demais colheitas ao longo do experimento. As diferenças entre as colheitas foram influenciadas pelo efeito sazonal, pois as alterações climáticas agem diretamente sobre o 
Tabela 3. Altura de planta $(\mathrm{cm})$, diâmetro de parte aérea $(\mathrm{cm})$, sobrevivência $(\%)$, massa fresca e seca de folhas e caules e, teor e rendimento de óleo essencial na produção de gerânio (P. graveolens), realizando colheitas a cada 16 semanas durante um período de um ano (plant height $(\mathrm{cm})$, canopy diameter $(\mathrm{cm})$, survival $(\%)$, fresh and dry weight of leaves and stems, and content and yield of essential oil production of geranium (P. graveolens) and harvests carried out every 8 weeks over a period of one year). São Cristóvão, UFS, 2011.

\begin{tabular}{|c|c|c|c|}
\hline \multirow{2}{*}{ Colheita } & \multicolumn{3}{|c|}{ Espaçamento (cm) } \\
\hline & $50 \times 50$ & $50 \times 60$ & $50 \times 80$ \\
\hline & \multicolumn{3}{|c|}{ Altura de planta (cm) } \\
\hline $1^{\mathrm{a}}$ & $69,31 \mathrm{aA}$ & $67,12 \mathrm{aA}$ & $56,17 \mathrm{bA}$ \\
\hline $2^{\mathrm{a}}$ & $76,75 \mathrm{aA}$ & $74,62 \mathrm{aA}$ & 73,16 aA \\
\hline $3^{\mathrm{a}}$ & $64,75 \mathrm{aB}$ & $59,33 \mathrm{aB}$ & $83,00 \mathrm{aA}$ \\
\hline \multirow[t]{2}{*}{$\underline{4^{\mathrm{a}}}$} & $30,16 \mathrm{bA}$ & $43,75 \mathrm{aA}$ & $52,00 \mathrm{bA}$ \\
\hline & \multicolumn{3}{|c|}{ Diâmetro de parte aérea $(\mathrm{cm})$} \\
\hline $1^{\mathrm{a}}$ & $70,96 \mathrm{aA}$ & $70,87 \mathrm{aA}$ & 66,68 aA \\
\hline $2^{\mathrm{a}}$ & $95,53 \mathrm{aA}$ & $91,66 \mathrm{aA}$ & $101,07 \mathrm{aA}$ \\
\hline $3^{\mathrm{a}}$ & $67,36 \mathrm{aB}$ & $79,00 \mathrm{aB}$ & $105,33 \mathrm{aA}$ \\
\hline \multirow[t]{2}{*}{$44^{\mathrm{a}}$} & $39,66 \mathrm{aB}$ & $70,50 \mathrm{aA}$ & $84,00 \mathrm{aA}$ \\
\hline & \multicolumn{3}{|c|}{ Sobrevivência (\%) } \\
\hline $1^{\mathrm{a}}$ & $100,00 \mathrm{aA}$ & $91,66 \mathrm{aA}$ & 91,66 aA \\
\hline $2^{\mathrm{a}}$ & $83,33 \mathrm{aA}$ & $83,33 \mathrm{aA}$ & 83,33 aA \\
\hline $3^{\mathrm{a}}$ & $75,00 \mathrm{aA}$ & $66,66 \mathrm{aA}$ & $50,00 \mathrm{bA}$ \\
\hline \multirow[t]{2}{*}{$\underline{4^{\mathrm{a}}}$} & $50,00 \mathrm{aA}$ & $25,00 \mathrm{bA}$ & $41,66 \mathrm{bA}$ \\
\hline & \multicolumn{3}{|c|}{ Massa fresca de folhas $\left(\mathrm{g} \mathrm{m}^{-2}\right)$} \\
\hline $1 \mathrm{a}$ & $730,00 \mathrm{aA}$ & $439,81 \mathrm{bB}$ & $513,89 \mathrm{aB}$ \\
\hline $2^{\mathrm{a}}$ & $674,04 \mathrm{aA}$ & $682,14 \mathrm{aA}$ & 594,72 aA \\
\hline $3^{\mathrm{a}}$ & $256,69 \mathrm{bA}$ & $149,28 \mathrm{cA}$ & $319,71 \mathrm{bA}$ \\
\hline \multirow[t]{2}{*}{$4^{\mathrm{a}}$} & $260,00 \mathrm{bA}$ & $241,67 \mathrm{cA}$ & $333,33 \mathrm{bA}$ \\
\hline & \multicolumn{3}{|c|}{ Massa seca de folhas $\left(\mathrm{g} \mathrm{m}^{-2}\right)$} \\
\hline $1^{\mathrm{a}}$ & $145,79 \mathrm{aA}$ & $87,59 \mathrm{bB}$ & $99,23 \mathrm{aB}$ \\
\hline $2^{\mathrm{a}}$ & $131,25 \mathrm{aA}$ & 133,88 aA & $119,12 \mathrm{aA}$ \\
\hline $3^{\mathrm{a}}$ & $56,02 \mathrm{bA}$ & $50,90 \mathrm{cA}$ & $67,83 \mathrm{bA}$ \\
\hline \multirow[t]{2}{*}{$\underline{4^{\mathrm{a}}}$} & $50,45 \mathrm{bA}$ & $29,74 \mathrm{cA}$ & $69,69 \mathrm{bA}$ \\
\hline & \multicolumn{3}{|c|}{ Massa fresca de caule $\left(\mathrm{g} \mathrm{m}^{-2}\right)$} \\
\hline 1 & $325,00 \mathrm{aA}$ & $204,63 \mathrm{bA}$ & $220,48 \mathrm{bA}$ \\
\hline $2^{\mathrm{a}}$ & $317,33 \mathrm{aA}$ & $379,52 \mathrm{aA}$ & $351,88 \mathrm{aA}$ \\
\hline $3^{\mathrm{a}}$ & $206,04 \mathrm{bA}$ & $121,27 \mathrm{bA}$ & $164,29 \mathrm{bA}$ \\
\hline \multirow[t]{2}{*}{$4^{\mathrm{a}}$} & $73,33 \mathrm{bA}$ & $83,33 \mathrm{bA}$ & $122,91 \mathrm{bA}$ \\
\hline & \multicolumn{3}{|c|}{ Massa seca de caule $\left(\mathrm{g} \mathrm{m}^{-2}\right)$} \\
\hline 1 & 50,38 aA & $33,96 \mathrm{bA}$ & $35,40 \mathrm{aA}$ \\
\hline $2^{\mathrm{a}}$ & $50,23 \mathrm{aA}$ & $60,53 \mathrm{aA}$ & $61,54 \mathrm{aA}$ \\
\hline $3^{\mathrm{a}}$ & $47,74 \mathrm{aA}$ & $25,92 \mathrm{bA}$ & 37,99 aA \\
\hline \multirow[t]{2}{*}{$4^{\mathrm{a}}$} & $13,42 \mathrm{bA}$ & $19,73 \mathrm{bA}$ & $30,30 \mathrm{aA}$ \\
\hline & \multicolumn{3}{|c|}{ Teor de óleo essencial (\%) } \\
\hline 1 & $1,33 \mathrm{aA}$ & $1,28 \mathrm{aA}$ & $1,31 \mathrm{aA}$ \\
\hline $2^{\mathrm{a}}$ & $1,36 \mathrm{aA}$ & $1,33 \mathrm{aA}$ & $1,32 \mathrm{aA}$ \\
\hline $3^{\mathrm{a}}$ & $0,97 \mathrm{aB}$ & $0,99 \mathrm{aB}$ & $1,58 \mathrm{aA}$ \\
\hline \multirow[t]{2}{*}{$4^{\mathrm{a}}$} & $1,34 \mathrm{aA}$ & $0,99 \mathrm{aA}$ & $1,17 \mathrm{aA}$ \\
\hline & \multicolumn{3}{|c|}{ Rendimento de óleo essencial $\left(\mathrm{mL} \mathrm{m}^{-2}\right)$} \\
\hline 1 & $1,90 \mathrm{aA}$ & $1,90 \mathrm{aA}$ & $1,29 \mathrm{aB}$ \\
\hline $2^{\mathrm{a}}$ & $1,79 \mathrm{aA}$ & $1,79 \mathrm{aA}$ & $1,61 \mathrm{aA}$ \\
\hline $3^{\mathrm{a}}$ & $0,58 \mathrm{bA}$ & $0,76 \mathrm{bA}$ & $1,03 \mathrm{aA}$ \\
\hline $4^{\mathrm{a}}$ & $0,76 \mathrm{bA}$ & $0,58 \mathrm{bA}$ & $0,80 \mathrm{aA}$ \\
\hline
\end{tabular}

Médias seguidas pelas mesmas letras, minúsculas nas colunas e maiúsculas nas linhas, não diferem entre si pelo teste de Scott-Knott (means followed by same letters, uppercase in lines and lowercase in columns do not differ by Scott-Knott test $)(p \leq 0,05)$. metabolismo da planta. Esses resultados são concordantes com alguns estudos que mostraram que espaçamentos menores entre linhas são mais rentáveis para o cultivo de gerânio (Rao, 2002; Ram et al., 2003; Bhan et al., 2006; Eiasu et al., 2009), embora ocorra ampla variação na adoção do espaçamento de plantio mais adequado ao cultivo do gerânio $(60 \times 30$, $60 \times 45,50 \times 50$ e $75 \times 45 \mathrm{~cm})$.

A colheita feita com 12 semanas apresentou diferenças apenas para diâmetro, sobrevivência, massa fresca e seca (folhas e caule) e rendimento de óleo essencial (Tabela 2). Com esse maior tempo entre as colheitas, houve um crescimento uniforme e, assim, não houve efeito sobre a altura das plantas.

$\mathrm{O}$ diâmetro de parte aérea para $50 \times 60 \mathrm{~cm}$ foi inferior aos demais espaçamentos nas seis primeiras colheitas (Tabela 2), mas proporcionou menor variação da taxa de sobrevivência (de $100 \%$ para $83,3 \%$ ). Ao contrário disso, nos espaçamentos 50x50 e 50x80 cm foram identificados altos índices de mortalidade de plantas nas duas últimas colheitas $\left(4^{\circ}\right.$ e $\left.5^{\circ}\right)$. Esses resultados podem ser devido à maior competição por luz e nutrientes e, podem ter proporcionado a maior mortalidade de plantas. Para o espaçamento $50 \times 80 \mathrm{~cm}$, isso pode ter sido agravado com a maior quantidade de plantas daninhas devido a esse maior espaçamento.

O espaçamento $50 \times 50 \mathrm{~cm}$ proporcionou maior rendimento de biomassa (massa fresca e seca de folhas e de caule) e de óleo essencial para o gerânio colhido em intervalo de 12 semanas em todas as colheitas, exceto na segunda (Tabela 2). Isso sugere que o adensamento das plantas no menor espaçamento promoveu menor desenvolvimento de plantas daninhas. Aliado a isso, o maior intervalo de tempo entre os cortes propiciou maior desenvolvimento vegetativo e, consequente, maior rendimento de biomassa e óleo essencial, concordando com os resultados de Rao (2002).

Quanto à colheita feita com 16 semanas, houve diferença significativa para todas as variáveis analisadas. Houve efeito dos espaçamentos apenas na terceira colheita, sendo superior o de $50 \times 80 \mathrm{~cm}$ (Tabela 3 ).Entre as colheitas, o espaçamento $50 \times 50 \mathrm{~cm}$ proporcionou $50 \%$ da altura que as demais colheitas. 
Tabela 4. Total de massa fresca e seca de folhas e de caules $\left(\mathrm{g} \mathrm{m}^{-2}\right)$ e rendimento de óleo essencial em função do intervalo de colheita e espaçamento na produção de gerânio $(P$. graveolens) durante o período de um ano (total fresh and dry weight of leaves and stems ( $\mathrm{g}$ $\mathrm{m}^{-2}$ ) and essential oil yield depending on the moment of harvest and spacing in the production of geranium (P. graveolens) during the period of one year). São Cristóvão, UFS, 2011.

\begin{tabular}{|c|c|c|c|}
\hline \multirow{2}{*}{ Intervalo de colheita } & \multicolumn{3}{|c|}{ Espaçamento (cm) } \\
\hline & $50 \times 50$ & $50 \times 60$ & $50 \times 80$ \\
\hline & \multicolumn{3}{|c|}{ Massa fresca de folhas $\left(\mathrm{g} \mathrm{m}^{-2}\right)$} \\
\hline 08 semanas & $2679,04 \mathrm{aA}$ & $2871,13 \mathrm{aA}$ & $1834,06 \mathrm{aA}$ \\
\hline 12 semanas & $1921,93 \mathrm{bA}$ & $1809,26 \mathrm{bA}$ & $1432,34 \mathrm{aA}$ \\
\hline \multirow[t]{2}{*}{16 semanas } & $1806,71 \mathrm{bA}$ & $2022,18 \mathrm{bA}$ & $1761,65 \mathrm{aA}$ \\
\hline & \multicolumn{3}{|c|}{ Massa seca de folhas $\left(\mathrm{g} \mathrm{m}^{-2}\right)$} \\
\hline 08 semanas & $424,62 \mathrm{aA}$ & $400,54 \mathrm{aA}$ & $277,04 \mathrm{aA}$ \\
\hline 12 semanas & $310,25 \mathrm{bA}$ & $266,96 \mathrm{bA}$ & $221,47 \mathrm{aA}$ \\
\hline \multirow[t]{2}{*}{16 semanas } & $279,09 \mathrm{bA}$ & $298,27 \mathrm{bA}$ & $218,35 \mathrm{aA}$ \\
\hline & \multicolumn{3}{|c|}{ Massa fresca de caule $\left(\mathrm{g} \mathrm{m}^{-2}\right)$} \\
\hline 08 semanas & $1035,08 \mathrm{aA}$ & $1165,60 \mathrm{aA}$ & $897,26 \mathrm{aA}$ \\
\hline 12 semanas & $701,91 \mathrm{bA}$ & $746,29 \mathrm{bA}$ & 760,99 aA \\
\hline \multirow[t]{2}{*}{16 semanas } & $662,06 \mathrm{bA}$ & $805,49 \mathrm{bA}$ & $859,58 \mathrm{aA}$ \\
\hline & \multicolumn{3}{|c|}{ Massa seca de caule $\left(\mathrm{g} \mathrm{m}^{-2}\right)$} \\
\hline 08 semanas & $136,85 \mathrm{aA}$ & $132,21 \mathrm{aA}$ & $100,61 \mathrm{aA}$ \\
\hline 12 semanas & $91,73 \mathrm{bA}$ & $85,64 \mathrm{bA}$ & $94,50 \mathrm{aA}$ \\
\hline \multirow[t]{2}{*}{16 semanas } & $80,75 \mathrm{bA}$ & $91,62 \mathrm{aA}$ & $96,94 \mathrm{aA}$ \\
\hline & \multicolumn{3}{|c|}{ Rendimento de óleo essencial $\left(\mathrm{mL} \mathrm{m}^{-2}\right)$} \\
\hline 08 semanas & $7,56 \mathrm{aA}$ & $7,49 \mathrm{aA}$ & $4,79 \mathrm{aA}$ \\
\hline 12 semanas & $4,74 \mathrm{bA}$ & $4,46 \mathrm{bA}$ & $3,43 \mathrm{aA}$ \\
\hline 16 semanas & $4,81 \mathrm{bA}$ & $5,30 \mathrm{bA}$ & $4,73 \mathrm{aA}$ \\
\hline
\end{tabular}

Médias seguidas pelas mesmas letras, minúsculas nas colunas e maiúsculas nas linhas, não diferem entre si pelo teste de Scott-Knott (means followed by same letters, uppercase in lines and lowercase in columns do not differ by Scott-Knott test $)(p \leq 0,05)$.

Com 50x80 cm maiores alturas foram obtidas na segunda $(73,16 \mathrm{~cm})$ e terceira $(83,00 \mathrm{~cm})$ colheitas (Tabela 3).

O diâmetro das plantas foi diferente apenas entre os espaçamentos nas duas ultimas colheitas, sendo 50x80 $\mathrm{cm}$ superior $(105,33 \mathrm{~cm})$ na terceira e $50 \times 50 \mathrm{~cm}$ inferior $(39,66 \mathrm{~cm})$ na quarta colheita (Tabela 3). O maior tempo para desenvolvimento das plantas, associado ao maior espaçamento entre linhas foi determinante para os ganhos em altura na colheita efetuada com 16 semanas.

A sobrevivência das plantas variou apenas entre as colheitas, sendo que a última colheita foi significativamente inferior $(25,0 \%)$ para $50 \times 60 \mathrm{~cm}$ e a terceira e quarta colheitas foram inferiores (50,0\% e $41,7 \%$, respectivamente) para o espaçamento de $50 \times 80 \mathrm{~cm}$. As plantas podem ter sido afetadas pelo maior
O gerânio (P. graveolens) demonstra potencial de rebrota dependente da estação ou período do ano em que se encontra, pois as condições climáticas do local de plantio e o estádio de desenvolvimento da planta interferem fortemente na produção de biomassa e óleo essencial. Fato evidenciado por estudo realizado em Rwanda, onde a realização de colheitas em períodos diferentes do dia resultou em significativa alteração na qualidade e no rendimento do óleo essencial de gerânio (Malatova et al., 2011).

Os resultados acima diferem do observado por May et al. (2008) que, estudando a influência do intervalo de cortes na produção de biomassa de duas espécies de capim limão (Cymbopogon citratus e Cymbopogon flexuosus), verificaram que, quanto maior o intervalo de tempo entre cortes, maior a produção de massa seca acumulada. Ou seja, quanto mais intenso é o intervalo de corte (intervalos entre cortes menores), menor o crescimento da planta, em razão do menor período de rebrota imposto.

Embora tenha havido muitas diferenças significativas em observação aos resultados individuais referentes a cada tempo de corte, os valores totais resumem o efeito geral dos tratamentos sobre o desenvolvimento do gerânio nas condições estabelecidas. O rendimento total de biomassa (massa fresca e seca de folhas e caules) e de óleo essencial de gerânio foi maior para as avaliações realizadas com o intervalo de corte de oito semanas, quando relacionado aos espaçamentos de $50 \times 50$ e $50 \times 60 \mathrm{~cm}$. Contudo, não houve diferenças significativas entre os intervalos de corte em relação ao espaçamento de 50×80 cm, nem entre os espaçamentos avaliados (Tabela 4).

Não foram evidenciadas diferenças significativas entre os espaçamentos testados quanto à produção de biomassa e óleo essencial; contudo, quanto aos intervalos de colheita, médias superiores foram registradas para o material colhido em intervalos de oito semanas. Assim, o menor espaçamento (50x50 $\mathrm{cm}$ ) e o menor intervalo de colheita (oito semanas) para a produção de biomassa e óleo essencial de gerânio proporcionaram melhores resultados nas condições 
do presente experimento.

\section{AGRADECIMENTOS}

Os autores agradecem à FAPITEC/ SE pelo financiamento da pesquisa, à CAPES pela bolsa de mestrado do segundo autor e ao CNPq, pelas bolsas de produtividade do primeiro e terceiro autores e pela bolsa de iniciação científica do quarto autor.

\section{REFERÊNCIAS}

BADI HN; YAZDANI D; ALI SM; NAZARI F. 2004. Effects of spacing and harvesting time on herbage yield and quality/quantity of oil in thyme, Thymus vulgaris L. Industrial Crops and Products 19: 231-236.

BAHN MK; DHAR AK; CHOUDHARY DK; REKHA K; BALYAN SS; KHAN S; AGARWAL SG; SHAWLAS. 2006. Geranium (Pelargonium sp. 'hybrid') essential oil in subtropical and temperate regions of Jammu and Kashmir. Flavour Fragrance Journal 21: $527-530$.

BERGO CL, MENDONÇA HA, SILVA MR. 2005. Efeito da época e freqüência de corte de pimenta longa (Piper hispidinervum $\mathrm{C}$. DC.) no rendimento de óleo essencial. Acta Amazonica 35: 111-117.

EIASU BK; STEYN JM; SOUNDY P. 2009. Rosescented geranium (Pelargonium capitatum $\mathrm{x} P$. radens) growth and essential oil yield response to different soil water depletion regimes. Agricultural Water Manager 96: 991-1000.

GOMES PB; MATA VG; RODRIGUES AE.
2004. Characterization of Portuguese-grown geranium oil (Pelargonium sp.). Journal of Essential oil Research 16: 490-495.

GUENTHER E. 1972. The Essential Oils 3: Individual essential oils of the plant families Rutaceae and Labiatae. Malabar: Krieger Publishing Company, 312 p.

KAUL PN; RAJESWARA RAO BR; BHATTACHARYA AK; MALLAVARAPU GR; RAMESH S. 1997. Changes in the chemical composition of rose-scented geranium 13 (Pelargonium sp) oil during storage. Journal of. Essential Oil Research. 9: 115-117.

KOTHARI SK; SINGH CP; SINGH K. 2002. Weed control in rose-scented geranium (Pelargonium spp). Pest Manager Science 58: 1254-1258.

LIS-BALCHIN M. 2002. History of nomenclature, usage and cultivation of Geranium and Pelargonium species. In: LIS-BALCHIN M. Geranium and Pelargonium: the genera Geranium and Pelargonium. New York: Taylor \& Francis, p. 5-10.

MALATOVA K; HITIMANA N; NIHYIBIZI T; SIMON J; JULIANI HR. 2011. Optimization of harvest regime and post-harvest handling in geranium production to maximize essential oil yield in Rwanda. Industrial Crops and Products. doi: 10.1016/j.indcrop.2010.12.018.

MAY A; BOVI OA; MAIA NB; MORAES ARA; PINHEIRO MQ; MARIO M. 2008. Influência do intervalo entre cortes sobre a produção de biomassa de duas espécies de capim limão. Horticultura Brasileira 26: 379-382.

MIGHRI H; AKROUT A; CASANOVA J; TOMI F; NEFFATI M. 2009. Impact of season and harvest frequency on biomass and essential oil yields of Artemisia Herba-Alba cultivated in southern Tunisia. Explication Agricultural 45: 499-508.
MISSAOUI AM; FASOULA VA; BOUTON JH. 2005. The effect of low plant density on response to selection for biomass production in switchgrass. Euphytica 142: 1-12.

PETERSON A; MACHMUDAH S; ROY BC; GOTO M; SASAKI M; HIROSE T. 2006. Extraction of essential oil from geranium (Pelargonium graveolens) with supercritical carbon dioxide. Journal of Chemical Technology Biotechnology 81: 167-172.

PURQUERIO LFV; DEMANT LAR; GOTO R; VILLAS BOAS RL. 2007. Efeito da adubação nitrogenada de cobertura e do espaçamento sobre a produção de rúcula. Horticultura Brasileira 25: 464-470.

RAM M; RAM D; ROY SK. 2003. Influence of an organic mulching on fertilizer nitrogen use efficiency and herb and essential oil yields in geranium (Pelargonium graveolens). Bioresource Technology 87: 273-278.

RAM M; SINGH R; NAQVI AA; KUMAR S. 1997. Effect of planting time on the yield and quality of essential oil in geranium Pelargonium graveolens. Journal of Horticultural Science 72: 807-810.

RANA VN; JUYAL JP; BLAZQUEZ MA. 2003. Chemical constituents of essential oil of Pelargonium graveolens leaves. International Journal of Aromatical Plants. 12: 216-218.

RAO BRR. 2002. Biomass yield, essential oil yield and essential oil composition of rose-scented geranium (Pelargonium species) as influenced by row spacings and intercropping with cornmint (Mentha arvensis L. f. piperascens Malinv. ex Holmes). Industrial Crops and Products. 16: 133-144.

SAXENA G; BANERJEE S; RAHMAN L.; MALLAVARAPU GR; SHARMAS; KUMAR S 2000. An efficient in vitro procedure for micropropagation and generation of somaclones of rose scented Pelargonium. Plant Science. 155: 133-140. 